\title{
Association of the Choline Acetyltransferase Gene with Responsiveness to Acetylcholinesterase Inhibitors in Alzheimer's Disease
}

Authors

Affiliations

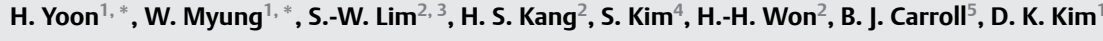

Affiliation addresses are listed at the end of the article
Key words

- Alzheimer's disease

- acetylcholinesterase inhibitors

- drug response

- CHAT

\section{Abstract}

$\nabla$

Introduction: The response to acetylcholinesterase inhibitors (AChEIs) of Alzheimer's disease (AD) patients varies depending on the genetic characteristics of the patient. We have examined the association of response to AChEIs and genetic polymorphisms in AD patients.

Methods: 158 patients with AD underwent treatment with AChEIs, and the therapeutic effect was assessed with the Korean version of the Mini Mental State Examination (K-MMSE). The asso-

\section{Introduction}

\section{$\nabla$}

Dementia is a progressive neurodegenerative disease with a rising prevalence and societal burden $[1,2]$. Alzheimer's disease (AD) is the most common form in dementia and it accounts for $60-70 \%$ of all cases [3].

$\mathrm{AD}$ is associated with widespread degeneration of cholinergic neurons, and acetylcholinesterase inhibitor (AChEI) drugs are approved for symptomatic treatment, with the aim of restoring the cholinergic deficit [4]. However, therapeutic response rates vary from $40-70 \%$ [5]. If the response to the drug initially selected is insufficient, a change of drugs can be considered. However, the recognition of non-response requires prolonged observation. Thus, an ability to predict response early in the course of $A D$ is an important therapeutic objective.

One promising approach is pharmacogenomics [6]. Several preliminary pharmacogenomic studies $[7,8]$ have reported that the clinical response to donepezil is highest in carriers of the APOE epsilon4 allele, although a recent large study obtained a negative result [9].

81 Irwon-ro

Gangnam-gu

Seoul 135-710

Korea

paulkim@skku.edu
${ }^{*}$ These individuals contributed equally to this article as co-first authors ciation of 25 SNPs located in 3 genes (CHAT, CHT and $A C H E$ ) with changes in the K-MMSE score was analyzed.

Results: The response to AChEIs in AD patients was significantly associated with 2 SNPs on the intronic region of CHAT rs2177370 (uncorrected $\mathrm{P}=0.0025$, FDR controlled $\mathrm{P}=0.026$ ) and rs3793790 (uncorrected $\mathrm{P}=0.0024$, FDR controlled $\mathrm{P}=0.026)$.

Conclusion: The results of our study confirmed again that genetic polymorphism of CHAT has an influence on drug response in AD.
The pathology of the brain cholinergic system is prominent in $\mathrm{AD}$ and $\mathrm{AChEI}$ drugs are widely used. Thus, the cholinergic system is a logical target for pharmacogenomic studies. There have been several studies on possible associations between genetic polymorphisms of cholinergicrelated genes and the therapeutic effect of AChEIs $[10,11]$. However, there are limitations as follows in those previous studies. First, the results are inconsistent. Second, the selection of SNPs was limited, so the entire candidate gene region was not covered. Moreover, ethnic heterogeneity was not explored in these studies. In the present study we have examined the polymorphic variations of the genes encoding 3 enzymes involved in the synthesis, transport, and metabolism of acetylcholine in the cholinergic system ( $\bullet$ Fig. 1) $[11,12]$. Choline acetyltransferase (ChAT) encoded by the gene CHAT synthesizes acetylcholine, using choline and acetyl-CoA as substrates [11]. The choline transporter (ChT) catalyzes the uptake of choline from the extracellular space to the neuronal cytoplasm, and is encoded by SLC5A7 [12]. Acetylcholine esterase (AChE) is encoded by $A C H E$ and acts to hydrolyze acetylcholine, thereby inactivating the neurotransmitter [11]. This study extends previous reports by the simultaneous coverage of 3 genes important for function of the brain cholinergic system. We 


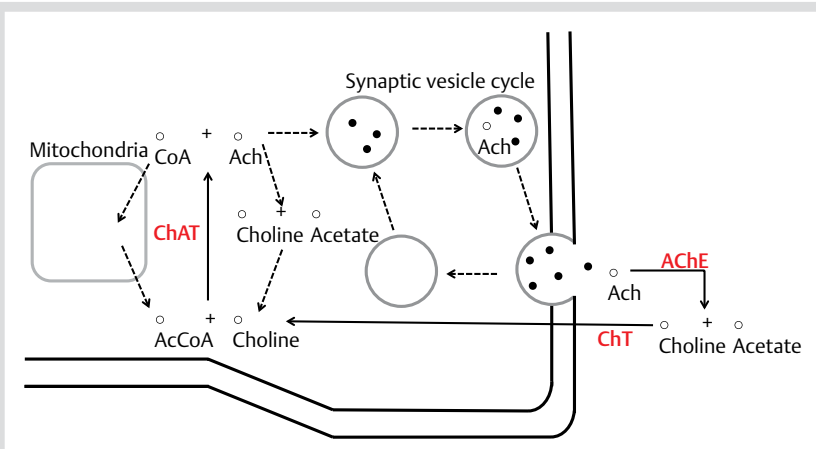

Fig. 1 The function of ChAT, AChE and ChT involved in synthesis and movement of acetylcholine in the cholinergic system. (from the KEGG database, http://www.genome.jp/kegg/). (Color figure available online only).

also aimed to assess in our Asian (Korean) population the replicability of previous reports in Caucasians $[10,11]$. The hypothesis of this exploratory study is that SNPs of genes involved in the synthesis and movement of acetylcholine may affect the response of AChEIs in $\mathrm{AD}$.

\section{Patients and Methods}

$\nabla$

\section{Subjects}

Subjects were 158 patients diagnosed with $\mathrm{AD}$ from the Clinical Trial Program in the Geropsychiatry Clinic at the Samsung Medical Center. All were of unrelated Korean ancestry. Patients were registered between November 2001 and January 2012. Subjects were eligible for this clinical trial only if they satisfied all following criteria: All patients were diagnosed as $A D$ or probable $A D$ according to the standards of the NINCDS-ADRDA (National Institute of Neurological and Communicative Disorders and StrokeAlzheimer's Disease and Related Disorders Association) [13]; They had a score of 26 or less in the Korean version of the MiniMental State Examination (K-MMSE) [14]; They had a history of cognitive decline which was gradual in onset and progressive for more than 6 months; they had a reliable caregiver who helped them to take their medication, participate in the assessment, and provide ongoing information about them [13]. Patients were excluded if any of the following conditions was present: other neurodegenerative diseases except AD (i.e., Parkinson's disease or Huntington's disease), psychiatric disorder or severe behavioral disturbances requiring psychotropic medications, cerebral injuries induced by trauma, hypoxia, and/or ischemia, clinically active cerebrovascular disease, medical history of seizure disorder, and other physical conditions requiring acute treatments.

All subjects underwent brain magnetic resonance imaging (MRI), neurological evaluation, and routine laboratory tests prior to this clinical trial in order to screen for other possible causes of dementia. The Institutional Review Board (IRB) at Samsung Medical Center approved the protocol. Written informed consent was obtained from both caregiver and patient. The study is registered (NCT01198093) in ClinicalTrials.gov.

\section{Procedures}

Subjects were assigned to receive monotherapy for 26 weeks with an acetylcholinesterase inhibitor (donepezil, galantamine or rivastigmine) as determined by a clinician. In this semi-naturalistic clinical trial, the choice of drug was based on the antici- pated side effects in at-risk individuals and on current clinical practice guidelines. Donepezil was administered to 84 patients, galantamine to 52 patients and rivastigmine to 22 patients.

Doses were titrated into the usual range based on tolerability and side effects. All subjects were assessed in clinic visits after 1 week and 4 weeks on drug to adjust the dosage and evaluate adverse events. Psychotropic medications except acetylcholinesterase inhibitors were not allowed with one exception. Benzodiazepines could be used only as a short-term adjunctive for insomnia. If the subjects did not show any significant changes or serious adverse events, the interval for clinic visits was increased to 13 weeks. Experienced geriatric psychiatrists performed the assessment at each visit for clinical review of cognitive status, to examine physical and neurological status, and to review adverse events. Vital sign checks, physical examinations, laboratory tests including complete blood counts, blood chemistry profiles, vitamin B12/folate levels, syphilis serology, thyroid function tests, and ECG at baseline were carried out in all subjects.

\section{Selection of SNP markers and genotyping}

These SNPs were genotyped using the MassARRAY system (Sequenom, Inc., San Diego, Calif). 25 SNPs were discovered and selected as candidate genes with the computer program Tagger [15] with criteria of $r^{2}>0.65$ and minor allele frequency $>0.05$ in combined Asian population (JPT/HCB). 21 for CHAT, 3 for SLC5A7 and one for $A C H E$ were genotyped. The total missing genotype counts were 50 (total call rate: $98.7 \%$ ), these genotyping data were not included in the SNP association analyses. All investigators and raters were blinded to the results of genotyping throughout the study. The laboratory worker who performed the genotyping was blind to clinical data of the subjects. The organization and selected SNP locations of CHAT gene are shown in $\odot$ Fig. 2 . There were no significant differences in genotype distribution of the 25 SNPs according to drug choice.

\section{Measures}

The response rate was assessed and compared at 26 weeks of treatment. Response was defined as no change (i.e., no deterioration) or improvement on the score of the Korean version of the Mini-Mental State Examination (K-MMSE) [16,17]. Global severity of disease was assessed according to the Clinical Dementia Rating (CDR) [18]. These research assessments of cognitive outcome were performed by a single, trained rater.

\section{Data analysis}

Continuous variables were presented as mean \pm standard deviation (SD) or as median and interquartile range. Categorical variables were summarized as frequencies and proportions. Wilcoxon rank-sum test or Student's t test was performed to compare continuous variables between 2 groups according to the normality of the distribution. The association of categorical variables was determined based on the chi-squared test in all subjects.

We assessed the associations between each SNP and responsiveness by using the exact Cochran-Armitage test for trend (a genotypic trend model) [19]. Chi-squared testing was used to examine deviation from Hardy-Weinberg equilibrium [20]. The four-gamete rule by Haploview was used to check linkage disequilibrium (LD) structure [21]. Phasing haplotypes were conducted using PHASE 2.1.1 for each of the haplotype blocks individually [22]. The exact Cochran-Armitage test for a trend was used to examine the associations between a haplotype 


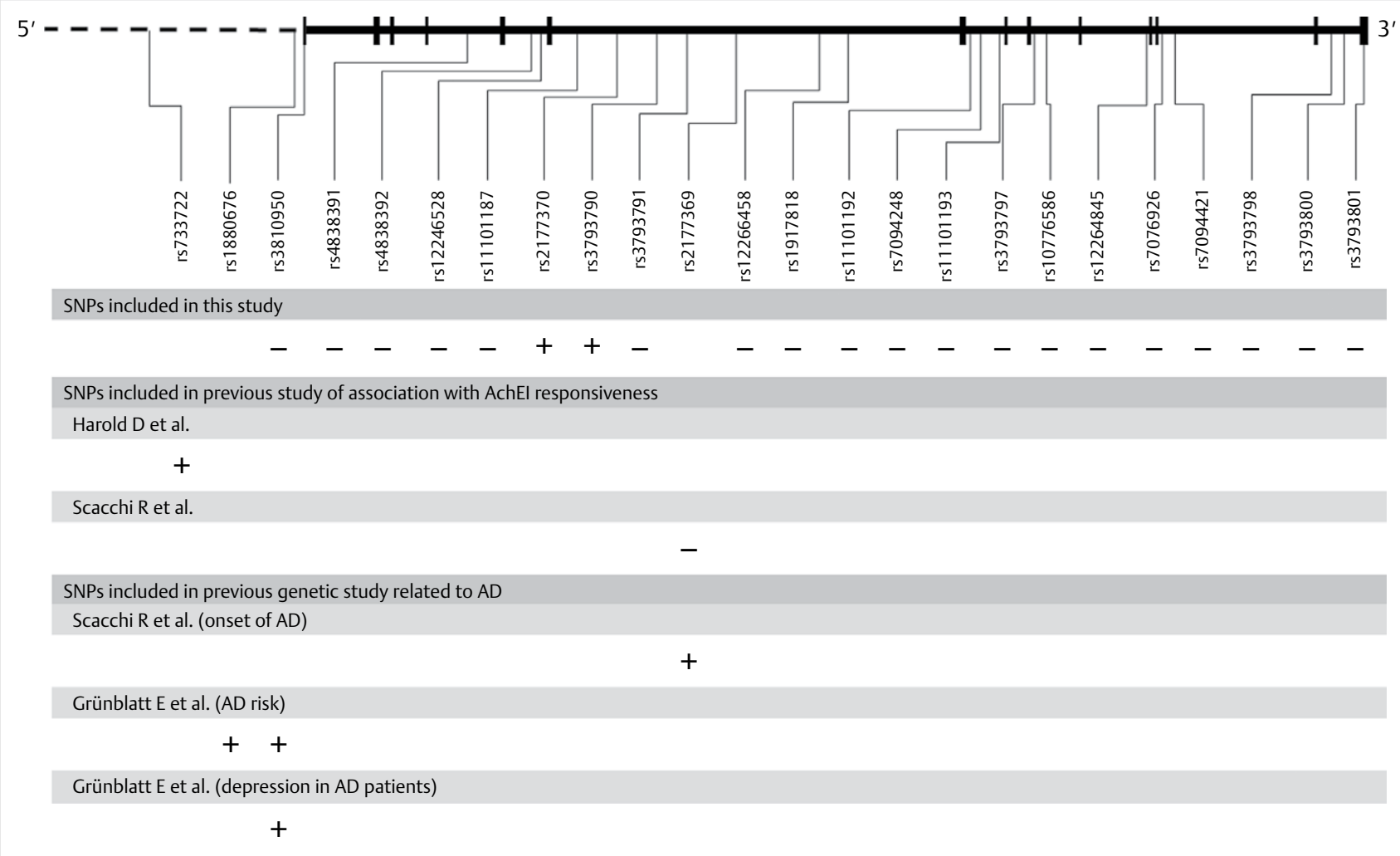

Fig. 2 CHAT organization and single-nucleotide polymorphism (SNP) locations (from National Center for Biotechnology Information Gene Database, http:// www.ncbi.nlm.nih.gov/gene/). The horizontal line represents the genomic sequence and vertical bars represent exons. Plus signs and minus signs denote SNPs with significant association and SNPs with negative results, respectively.

Table 1 Clinical and demographic characteristics $(n=158)$.

\begin{tabular}{|c|c|c|c|c|c|}
\hline & Total & Responder $(n=102)$ & Non-Responder $(n=56)$ & Statistics & $\mathbf{P}$ \\
\hline Gender, male (\%) & $64(40.5 \%)$ & $39(38.2 \%)$ & $25(44.6 \%)$ & $\mathrm{X}^{2}{ }_{1}=0.62$ & $0.43^{\mathrm{a}}$ \\
\hline Age (year, mean $\pm S D)$ & $72.66 \pm 8.31$ & $73.47 \pm 8.18$ & $71.18 \pm 8.41$ & $t_{156}=-1.67$ & $0.10^{\mathrm{b}}$ \\
\hline Education (year, median and interquartile) & $8(6,12)$ & $6(6,12)$ & $9(6,12)$ & $Z=1.19$ & $0.23^{c}$ \\
\hline \multicolumn{6}{|l|}{ Drug (\%) } \\
\hline Donepezil & $84(53.2 \%)$ & $57(55.9 \%)$ & $27(48.2 \%)$ & \multirow{3}{*}{$X^{2}{ }_{2}=1.39$} & \multirow{3}{*}{$0.50^{\mathrm{a}}$} \\
\hline Galantamine & $52(32.9 \%)$ & $33(32.4 \%)$ & $19(33.9 \%)$ & & \\
\hline Rivastigmine & $22(13.9 \%)$ & $12(11.8 \%)$ & $10(17.9 \%)$ & & \\
\hline \multicolumn{6}{|l|}{ Baseline Dementia Severity } \\
\hline K-MMSE score (mean \pm SD) & $19.11 \pm 4.73$ & $18.55 \pm 4.70$ & $20.13 \pm 4.64$ & $t_{156}=2.02$ & $0.04^{b}$ \\
\hline \multicolumn{6}{|l|}{$\operatorname{CDR}(\%)$} \\
\hline 0.5 & $54(34.2 \%)$ & $32(31.4 \%)$ & 22 (39.3\%) & \multirow{3}{*}{$X^{2}{ }_{2}=1.30$} & \multirow{3}{*}{$0.52^{\mathrm{a}}$} \\
\hline 1 & $74(46.8 \%)$ & $51(50.0 \%)$ & $23(41.1 \%)$ & & \\
\hline 2 & $30(19.0 \%)$ & $19(18.6 \%)$ & $11(19.6 \%)$ & & \\
\hline
\end{tabular}

SD, standard deviation; K-MMSE score, Korean Mini Mental State Examination score; CDR, Clinical Dementia Rating

a. Chi-squared test was used; b. Student's t test was used; c. Wilcoxon rank-sum test was used

allele and response. For the significance of association of a SNP or haplotype allele, the false discovery rate (FDR) control was used to correct each P-value [23].

The associated SNPs and haplotype alleles were entered into a multiple logistic regression model to evaluate the impact of each genetic variable on response, adjusting for other variables. In this model, the genetic variable represented the minor allele count for a subject $(0,1$ or 2$)$ and the dependent variable represented the treatment outcome ( $1=$ response and $0=$ nonresponse). Results were considered as significant with a threshold of $\mathrm{P}<0.05$. All statistical tests were performed using SAS 9.1 (SAS Institute, Inc., Cary, North Carolina).

\section{Results}

\section{Subject characteristics}

Clinical and demographic characteristics are shown in $\odot$ Table 1 . Mean age of the subjects was $72.66(\mathrm{SD}=8.31)$ years and most were in the early stage of Alzheimer's disease. The rate of response to acetylcholinesterase inhibitors was 102 of 158 (64.6\%). There was no significant difference between responders and non-responders with respect to gender, age, education level and baseline global severity (CDR). The rate of response was not affected by choice of drug (donepezil, galantamine and rivastigmine). However, there was a marginally significant difference 
Table 2 SNP association analysis with responsiveness.

\begin{tabular}{|c|c|c|c|c|c|c|c|}
\hline \multicolumn{2}{|c|}{ SNP by Group } & \multicolumn{2}{|c|}{ Genotype Count } & \multirow{2}{*}{ Location $^{\text {a. }}$} & \multirow[t]{2}{*}{ Statistics for HWE ${ }^{\text {b. }}$} & \multirow[t]{2}{*}{$\mathbf{P}^{c .}$} & \multirow[t]{2}{*}{ FDR Corrected P } \\
\hline & & & & & & & \\
\hline rs3810950 & GG & GA & $\mathrm{AA}$ & & & & \\
\hline Responder & 75 & 24 & 1 & 50824619 & $X^{2}{ }_{1}=0.34$ & 0.73 & 1 \\
\hline Non-Responder & 40 & 14 & 1 & & $P=0.56$ & & \\
\hline rs4838391 & $\mathrm{CC}$ & TC & TT & & & & \\
\hline Responder & 58 & 39 & 4 & 50832109 & $X^{2}{ }_{1}=0.09$ & 0.14 & 1 \\
\hline Non-Responder & 27 & 23 & 6 & & $P=0.77$ & & \\
\hline rs4838392 & $\mathrm{AA}$ & GA & GG & & & & \\
\hline Responder & 34 & 49 & 15 & 50834978 & $X^{2}{ }_{1}=0.46$ & 0.61 & 1 \\
\hline Non-Responder & 19 & 26 & 6 & & $P=0.50$ & & \\
\hline rs12246528 & GA & GG & $\mathrm{AA}$ & & & & \\
\hline Responder & 12 & 89 & 0 & 50835264 & $X^{2}{ }_{1}=103.35$ & 0.42 & 1 \\
\hline Non-Responder & 4 & 51 & 0 & & $P=2.80 \times 10^{-24}$ & & \\
\hline rs11101187 & $\mathrm{CC}$ & CT & TT & & & & \\
\hline Responder & 93 & 9 & 0 & 50837034 & $X^{2}{ }_{1}=2.18$ & 0.79 & 1 \\
\hline Non-Responder & 53 & 2 & 1 & & $P=0.14$ & & \\
\hline rs2177370 & $\mathrm{CC}$ & TC & TT & & & & \\
\hline Responder & 48 & 47 & 6 & 50838874 & $X^{2}{ }_{1}=0.29$ & 0.003 & 0.03 \\
\hline Non-Responder & 44 & 7 & 4 & & $P=0.59$ & & \\
\hline rs3793790 & $\mathrm{AA}$ & GA & GG & & & & \\
\hline Responder & 46 & 52 & 4 & 50840736 & $X^{2}{ }_{1}=0.91$ & 0.002 & 0.03 \\
\hline Non-Responder & 42 & 10 & 3 & & $P=0.34$ & & \\
\hline rs3793791 & $\mathrm{CC}$ & TC & CC & & & & \\
\hline Responder & 49 & 45 & 8 & 50841704 & $X^{2}{ }_{1}=0.25$ & 0.90 & 1 \\
\hline Non-Responder & 31 & 18 & 7 & & $P=0.61$ & & \\
\hline rs12266458 & $\mathrm{CC}$ & TC & TT & & & & \\
\hline Responder & 36 & 47 & 19 & 50847997 & $X^{2}{ }_{1}=0.81$ & 0.21 & 1 \\
\hline Non-Responder & 15 & 25 & 15 & & $P=0.37$ & & \\
\hline rs1917818 & $\mathrm{AA}$ & CA & CC & & & & \\
\hline Responder & 62 & 30 & 10 & 50849342 & $X^{2}{ }_{1}=6.36$ & 0.20 & 1 \\
\hline Non-Responder & 39 & 13 & 3 & & $P=0.01$ & & \\
\hline rs11101192 & GG & GA & $\mathrm{AA}$ & & & & \\
\hline Responder & 60 & 32 & 8 & 50854767 & $X^{2}{ }_{1}=4.66$ & 1 & 1 \\
\hline Non-Responder & 34 & 15 & 6 & & $P=0.03$ & & \\
\hline rs7094248 & $\mathrm{CC}$ & GC & GG & & & & \\
\hline Responder & 52 & 36 & 12 & 50855368 & $X^{2}{ }_{1}=4.34$ & 0.55 & 1 \\
\hline Non-Responder & 33 & 17 & 6 & & $P=0.04$ & & \\
\hline rs11101193 & GG & GT & TT & & & & \\
\hline Responder & 71 & 24 & 7 & 50856138 & $X^{2}{ }_{1}=7.56$ & 0.68 & 1 \\
\hline Non-Responder & 41 & 12 & 3 & & $P=0.01$ & & \\
\hline rs3793797 & $\mathrm{TT}$ & CT & CC & & & & \\
\hline Responder & 64 & 27 & 11 & 50857849 & $X^{2}{ }_{1}=3.15$ & 1 & 1 \\
\hline Non-Responder & 31 & 22 & 2 & & $P=0.08$ & & \\
\hline rs10776586 & $\mathrm{TT}$ & TC & CC & & & & \\
\hline Responder & 53 & 36 & 8 & 50858346 & $X^{2}{ }_{1}=0.08$ & 0.89 & 1 \\
\hline Non-Responder & 27 & 20 & 3 & & $P=0.78$ & & \\
\hline rs12264845 & $\mathrm{CC}$ & CA & $\mathrm{AA}$ & & & & \\
\hline Responder & 37 & 54 & 11 & 50863083 & $X^{2}{ }_{1}=0.69$ & 0.90 & 1 \\
\hline Non-Responder & 23 & 24 & 8 & & $P=0.41$ & & \\
\hline rs7076926 & $\mathrm{TT}$ & CT & CC & & & & \\
\hline Responder & 55 & 43 & 4 & 50863565 & $X^{2}{ }_{1}=1.42$ & 0.49 & 1 \\
\hline Non-Responder & 28 & 24 & 4 & & $P=0.23$ & & \\
\hline rs7094421 & $\mathrm{AA}$ & GA & GG & & & & \\
\hline Responder & 76 & 23 & 1 & 50863623 & $X^{2}{ }_{1}=0.49$ & 0.25 & 1 \\
\hline Non-Responder & 47 & 9 & 0 & & $P=0.48$ & & \\
\hline rs3793798 & $\mathrm{TT}$ & AT & $\mathrm{AA}$ & & & & \\
\hline Responder & 51 & 39 & 10 & 50871466 & $X^{2}{ }_{1}=0.21$ & 0.53 & 1 \\
\hline Non-Responder & 25 & 25 & 6 & & $P=0.64$ & & \\
\hline rs3793800 & $\mathrm{AA}$ & AG & GG & & & & \\
\hline Responder & 80 & 21 & 1 & 50871716 & $X^{2}{ }_{1}=0.29$ & 0.43 & 1 \\
\hline Non-Responder & 47 & 9 & 0 & & $P=0.59$ & & \\
\hline rs3793801 & $\mathrm{CC}$ & TC & TT & & & & \\
\hline Responder & 44 & 47 & 10 & 50872912 & $X^{2}{ }_{1}=0.67$ & 0.61 & 1 \\
\hline Non-Responder & 26 & 26 & 4 & & $P=0.41$ & & \\
\hline
\end{tabular}




\section{Table 2 Continued.}

\begin{tabular}{|c|c|c|c|c|c|c|c|}
\hline SNP by Group & & \multicolumn{2}{|c|}{ Genotype Count } & Location $^{\text {a. }}$ & Statistics for HWE ${ }^{b}$. & Pc. & FDR Corrected P \\
\hline \multicolumn{8}{|c|}{ SLC5A7 (chromosome 2) } \\
\hline rs6542746 & CC & $\mathrm{TC}$ & TT & & & & \\
\hline Responder & 50 & 41 & 10 & 13279665 & $X^{2}{ }_{1}=0.02$ & 0.37 & 1 \\
\hline Non-Responder & 31 & 22 & 3 & & $P=0.88$ & & \\
\hline rs6720783 & GG & GT & TT & & & & \\
\hline Responder & 48 & 46 & 6 & 13297151 & $X^{2}{ }_{1}=2.64$ & 1 & 1 \\
\hline Non-Responder & 25 & 26 & 3 & & $P=0.11$ & & \\
\hline rs11685873 & GG & AG & AA & & & & \\
\hline Responder & 73 & 27 & 1 & 13285348 & $X^{2}{ }_{1}=0.86$ & 0.75 & 1 \\
\hline Non-Responder & 42 & 10 & 4 & & $P=0.35$ & & \\
\hline \multicolumn{8}{|c|}{ ACHE (chromosome 7) } \\
\hline rs6942609 & GG & AG & AA & & & & \\
\hline Responder & 40 & 50 & 11 & 38928323 & $X^{2}{ }_{1}=1.06$ & 0.70 & 1 \\
\hline Non-Responder & 24 & 27 & 5 & & $P=0.30$ & & \\
\hline
\end{tabular}

SNP, single-nucleotide polymorphism; HWE, Hardy-Weinberg equilibrium; FDR, false discovery rate

a. Genomic position (NCBI Build 37)

b. Chi-squared test was used

c. Exact Cochran-Armitage test for trend was used

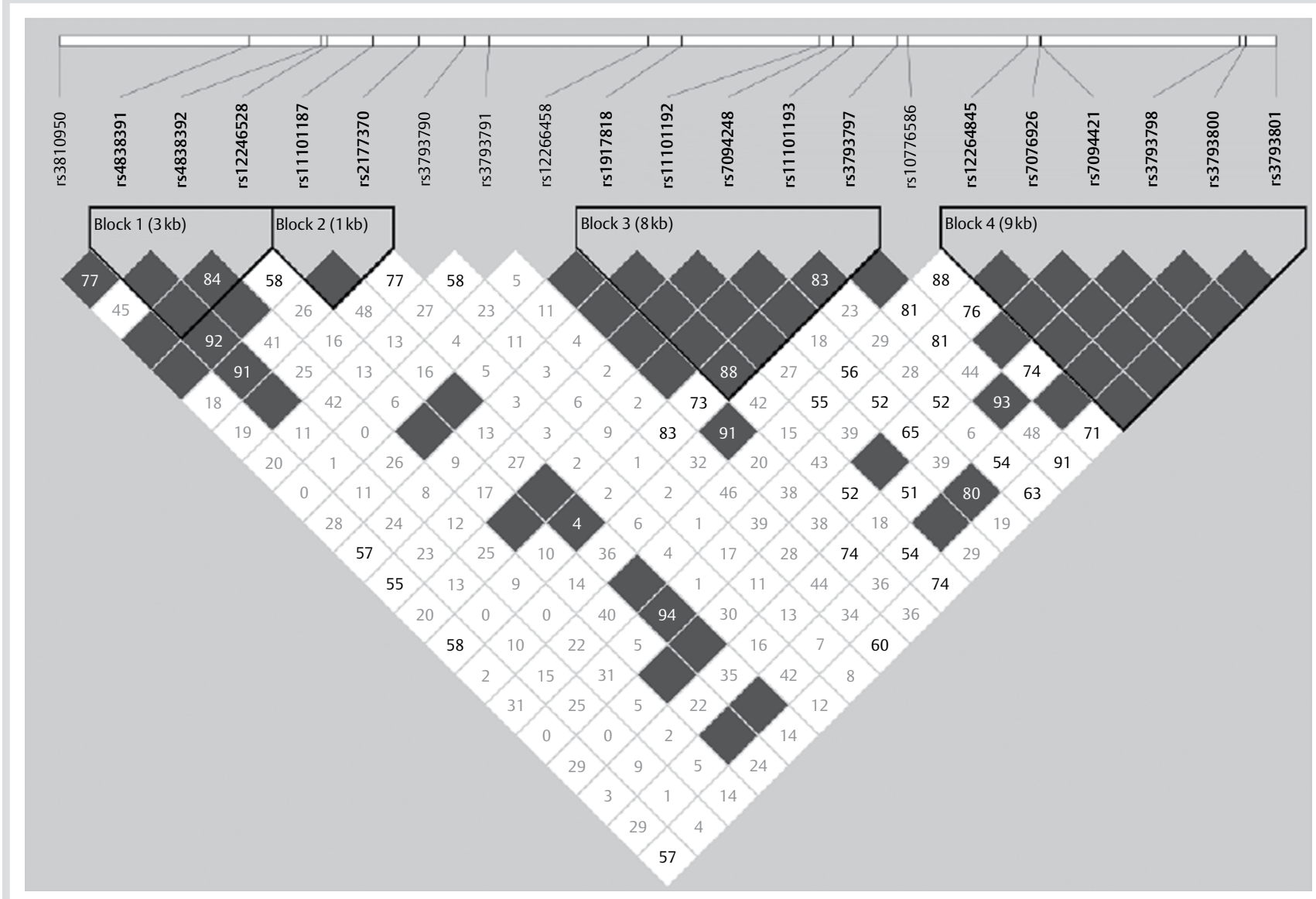

Fig. 3 Linkage disequilibrium (LD) and haplotype structure of CHAT. Pairwise SNP $\left|D^{\prime}\right|$ values $(\times 100)$ of linkage ( $\left|D^{\prime}\right|=1$ not shown) are shown together with haplotype blocks. Black squares represent less than 4 distinct 2-marker haplotypes and white squares represent 4 distinct 2-marker haplotypes by the 4 gamete rule. Triangles surrounding the markers represent haplotype blocks identified using the default 4-gamete rule algorithm of Haploview 4.2.

between responders and non-responders in baseline K-MMSE score $(P=0.04)$.
SNP association analysis with responder of acetylcholinesterase inhibitors

The results of SNP association analysis are shown in $\odot$ Table 2. The observed genotype frequencies in each case fitted the ones expected according to the Hardy-Weinberg equilibrium, except one SNP, rs12246528 $\left(\mathrm{P}=2.80 \times 10^{-24}\right)$. However, we did not 
exclude this SNP, because this pharmacogenetic study was conducted in AD patients and did not have a normal control group $[19,24]$. Moreover, 2 adjacent SNPs (rs2177370 and rs3793790) were significantly associated with response.

The rs2177370 in the intronic region of CHAT gene was significantly associated with response (uncorrected $\mathrm{P}=0.0025$, FDR controlled $\mathrm{P}=0.026$ ). The rs3793790 located in the same intron of the rs2177370 showed a significant association with responsiveness (uncorrected $\mathrm{P}=0.0024$, FDR controlled $\mathrm{P}=0.026$ ). These associations were preserved after controlling for gender, age, education year, drug and baseline K-MMSE score (for rs2177370, $\mathrm{P}=0.0065$, odds ratio $=2.45,95 \%$ confidence interval $=1.28-4.68$; for rs3793790, $\mathrm{P}=0.0039$, odds ratio $=2.73,95 \%$ confidence interval $=1.38-5.38$ ).

\section{Haplotype association analysis with responder of acetylcholinesterase inhibitors}

We discovered 4 haplotype blocks in the CHAT gene ( $\odot$ Fig. 3). Among the 13 haplotype allele, 2 alleles in block 2 that included rs2177370 had significant associations with response (for haplotype $C C$, uncorrected $\mathrm{P}=0.004$, FDR controlled $\mathrm{P}=0.023$; for haplotype $\mathrm{CT}$, uncorrected $\mathrm{P}=0.003$, FDR controlled $\mathrm{P}=0.023$ ). These haplotypes were also associated with response after controlling for gender, age, education year, drug and baseline K-MMSE score (for haplotype CC, $\mathrm{P}=0.006$, odds ratio $=0.44,95 \%$ confidence interval $=0.24-0.79$; for haplotype $\mathrm{CT}, \mathrm{P}=0.006$, odds ratio $=2.47$, $95 \%$ confidence interval=1.29-4.72). However, no haplotype blocks were found to be significantly associated with response in the SLC5A7 gene or the ACHE gene ( $\odot$ Table 3 ).

\section{Discussion}

In this study we assessed 25 SNPs of 3 cholinergic system genes (CHAT, SLC5A7 and ACHE) for association with response to AChEI drugs in $\mathrm{AD}$. We found that 2 SNPs in the intronic region of $C H A T$, rs2177370 and rs3793790 had a significant association with drug response. Haplotype association analysis which was additionally performed showed that block 2 including rs2177370 among 4 haplotype blocks of the CHAT gene had a significant association with drug response. However, the ACHE and SLC5A7 genes did not contain SNPs or haplotypes that were significantly associated with response. From this we conclude that the brain's ability to synthesize ACh in AD is a critical factor for response to AChEIs, whereas transport and inactivation of the transmitter are less important factors.

The association of CHAT gene polymorphisms with response is consistent with a previous study [10], but the association with CHAT rs2177370 has not been previously described. In one previous study CHAT rs733722 had a significant association with AChEI drug response in AD patients [10]. In a second study, no association of CHAT rs2177369 with response was reported [11]. The CHAT gene has also been studied for association with AD onset [11], AD risk factor [25], and depression in AD [25] (○ Fig. 2). Although the significantly associated SNPs in our study differ from those in previous studies, these other results suggest convergent evidence for the importance of the CHAT gene as a significant gene marker that affects the response of AChEIs. Our results call attention to the role of ChAT in the synthesis of acetylcholine and to the mechanism of action of AChEIs in patients with $\mathrm{AD}$.
Table 3 Haplotype association analysis with responsiveness in CHAT gene.

\begin{tabular}{|c|c|c|c|c|c|}
\hline \multirow[t]{2}{*}{ Haplotype by Group } & \multicolumn{3}{|c|}{ Allele count } & \multirow[t]{2}{*}{$\mathbf{p}^{\mathbf{a}}$} & \multirow[t]{2}{*}{ FDR Corrected P } \\
\hline & 0 & 1 & 2 & & \\
\hline \multicolumn{6}{|c|}{ Block 1 (rs4838391-rs4838392-rs12246528) } \\
\hline \multicolumn{6}{|c|}{ CGG } \\
\hline Responder & 44 & 46 & 12 & 0.70 & 0.83 \\
\hline Non-Responder & 23 & 30 & 3 & & \\
\hline \multicolumn{6}{|l|}{ CAG } \\
\hline Responder & 41 & 48 & 13 & 0.61 & 0.83 \\
\hline Non-Responder & 23 & 29 & 4 & & \\
\hline \multicolumn{6}{|l|}{ TAG } \\
\hline Responder & 58 & 40 & 4 & 0.14 & 0.45 \\
\hline Non-Responder & 27 & 23 & 6 & & \\
\hline \multicolumn{6}{|c|}{ Block 2 (rs11101187-rs2177370) } \\
\hline \multicolumn{6}{|l|}{ CC } \\
\hline Responder & 10 & 48 & 44 & 0.004 & 0.023 \\
\hline Non-Responder & 5 & 9 & 42 & & \\
\hline \multicolumn{6}{|l|}{ CT } \\
\hline Responder & 49 & 47 & 6 & 0.003 & 0.023 \\
\hline Non-Responder & 45 & 7 & 4 & & \\
\hline \multicolumn{6}{|c|}{ Block 3 (rs1917818-rs11101192-rs7094248-rs11101193-rs3793797) } \\
\hline \multicolumn{6}{|c|}{ CGCTT } \\
\hline Responder & 72 & 23 & 7 & 0.78 & 0.85 \\
\hline Non-Responder & 41 & 12 & 3 & & \\
\hline \multicolumn{6}{|l|}{ AGCGC } \\
\hline Responder & 64 & 28 & 10 & 1 & 1 \\
\hline Non-Responder & 31 & 23 & 2 & & \\
\hline \multicolumn{6}{|l|}{ AAGGT } \\
\hline Responder & 61 & 32 & 9 & 0.62 & 0.83 \\
\hline Non-Responder & 34 & 16 & 6 & & \\
\hline \multicolumn{6}{|l|}{ AGCGT } \\
\hline Responder & 60 & 38 & 4 & 0.07 & 0.32 \\
\hline Non-Responder & 25 & 26 & 5 & & \\
\hline \multicolumn{6}{|c|}{$\begin{array}{l}\text { Block } 4 \text { (rs12264845-rs7076926-rs7094421-rs3793798-rs3793800- } \\
\text { rs3793801) }\end{array}$} \\
\hline \multicolumn{6}{|c|}{ ATGTGC } \\
\hline Responder & 80 & 21 & 1 & 0.43 & 0.83 \\
\hline Non-Responder & 47 & 9 & 0 & & \\
\hline \multicolumn{6}{|l|}{ ACATAC } \\
\hline Responder & 55 & 43 & 4 & 0.49 & 0.83 \\
\hline Non-Responder & 28 & 24 & 4 & & \\
\hline \multicolumn{6}{|l|}{ CTAAAC } \\
\hline Responder & 51 & 41 & 10 & 0.62 & 0.83 \\
\hline Non-Responder & 25 & 25 & 6 & & \\
\hline \multicolumn{6}{|l|}{ CTATAT } \\
\hline Responder & 45 & 47 & 10 & 0.70 & 0.83 \\
\hline Non-Responder & 26 & 26 & 4 & & \\
\hline
\end{tabular}

Acetylcholinesterase inhibitors are drugs that inhibit the acetylcholinesterase enzyme from breaking down acetylcholine, thereby increasing both the level and duration of action of the neurotransmitter acetylcholine [26]. However, AChEI drugs depend for their efficacy on an adequate synthesis of ACh. When ACh synthesis already is impaired by degeneration of cholinergic neurons in $\mathrm{AD}$, then a genetically determined relatively high synthesis capacity in the remaining neurons would be expected to favor response to AChEI drugs, and vice versa. We might infer that haplotype CC, with an odds ratio for response of 0.44 , is associated with a relatively reduced rate of ACh synthesis, whereas haplotype CT, with an OR of 2.47 , is associated with a relatively high rate of $\mathrm{ACh}$ synthesis. Additional studies are needed to establish the functional direction of influence that rs2177370 and rs3793790 exert on the activity of CHAT. 
We did not confirm the report of Scacchi et al. that the ACHE rs2571598 had a significant association with drug response in $A D$ patients treated with rivastigmine [11]. We found no association between the ACHE gene and response to AChEI drugs. The discrepancies between our study and Scacchi's may due to differences of SNP selection, ethnicity and the genetic models adopted. In a previous study examining the CHAT gene, rs3810950 had a significant association with both depression [25] and disease progression [27] in AD. However, there was no association with drug response in this study. Because both comorbid depression and disease stage can influence cognitive function in $A D$, these factors will need to be considered in future pharmacogenetic studies.

We conducted this study in Korean patients. In our previous pharmacogenetic study of the serotonin transporter in patients with depression, conflicting results were reported according to ethnicity (Caucasian, Asian) [28]. Most of the existing genetic studies for drug response in $\mathrm{AD}$ patients have been limited to Caucasian populations. Thus, replication studies in different ethnic populations will be required. Our study is the first haplotype association study of response of AChEIs and found that haplotype blocks located on CHAT may affect response in both favorable and unfavorable directions. A possible limitation or a potential advantage in this study was the use of 3 members of the AChEI drug class. On the one hand we lack statistical power to examine SNP associations with response to individual drugs. On the other hand, by using all the common members of the AChEI class our results may be generalizable to the clinical setting.

\section{Acknowledgements}

$\nabla$

This study was supported by grants of the Korea Health 21 R\&D Project, Ministry of Health, Welfare and Family Affairs, Korea (A050079 and A060618) and Eisai Korea.

\section{Author Contributions}

\section{$\nabla$}

The individual authors contributed as follows: Doh Kwan Kim, Woojae Myung, Shin-Won Lim and Hyeyeon Yoon were involved in study planning and the writing of the manuscript; Doh Kwan Kim conducted the clinical parts of the study. Hyo Shin Kang was involved in data acquisition, Seonwoo Kim, Woojae Myung, Hong-Hee Won and Hyeyeon Yoon performed the statistical analyses; Bernard J. Carroll edited the manuscript and assisted with interpretation of the data.

\section{Conflict of Interests}

$\nabla$

The author(s) declared no potential conflicts of interest with respect to the research, authorship, and/or publication of this article.

\footnotetext{
Affiliations

${ }^{1}$ Department of Psychiatry, Samsung Medical Center, Sungkyunkwan

University School of Medicine, Seoul, Korea

${ }^{2}$ Center for Clinical Research, Samsung Biomedical Research Institute, Seoul, Korea

${ }^{3}$ SAIHST, Sungkyunkwan University School of Medicine, Seoul, Korea

${ }^{4}$ Biostatistics Team, Samsung Biomedical Research Institute, Seoul, Korea

${ }^{5}$ Pacific Behavioral Research Foundation, Carmel, CA, USA
}

\section{References}

1 Kawas $\mathrm{CH}$, Brookmeyer $\mathrm{R}$. Aging and the public health effects of dementia. N Engl J Med 2001; 344: 1160-1161

2 Ballard C, Gauthier S, Corbett A et al Alzheimer's disease. Lancet 2011; 377: 1019-1031

3 Hendrie HC. Epidemiology of dementia and Alzheimer's disease. Am J Geriatr Psychiatry 1998; 6: S3-S18

4 Ellis JM. Cholinesterase inhibitors in the treatment of dementia. J Am Osteopath Assoc 2005; 105: 145-158

5 Jann MW, Shirley KL, Small GW. Clinical pharmacokinetics and pharmacodynamics of cholinesterase inhibitors. Clin Pharmacokinet 2002; 41: 719-739

6 Scott SA. Personalizing medicine with clinical pharmacogenetics. Genet Med 2011; 13: 987-995

7 Bizzarro A, Marra C, Acciarri A et al. Apolipoprotein E epsilon4 allele differentiates the clinical response to donepezil in Alzheimer's disease. Dement Geriatr Cogn Disord 2005; 20: 254-261

8 Choi SH, Kim SY, Na HR et al. Effect of ApoE genotype on response to donepezil in patients with Alzheimer's disease. Dement Geriatr Cogn Disord 2008; 25: 445-450

9 Rigaud AS, Traykov L, Latour F et al. Presence or absence of at least one epsilon 4 allele and gender are not predictive for the response to donepezil treatment in Alzheimer's disease. Pharmacogenetics 2002; 12: $415-420$

10 Harold D, Macgregor S, Patterson CE et al. A single nucleotide polymorphism in CHAT influences response to acetylcholinesterase inhibitors in Alzheimer's disease. Pharmacogenet Genomics 2006; 16: 75-77

11 Scacchi R, Gambina G, Moretto $G$ et al. Variability of AChE, BChE, and ChAT genes in the late-onset form of Alzheimer's disease and relationships with response to treatment with Donepezil and Rivastigmine. Am J Med Genet B Neuropsychiatr Genet 2009; 150B: 502-507

12 Brandon EP, Mellott T, Pizzo DP et al. Choline transporter 1 maintains cholinergic function in choline acetyltransferase haploinsufficiency. J Neurosci 2004; 24: 5459-5466

13 McKhann G, Drachman D, Folstein $M$ et al. Clinical diagnosis of Alzheimer's disease: report of the NINCDS-ADRDA Work Group under the auspices of Department of Health and Human Services Task Force on Alzheimer's Disease. Neurology 1984; 34: 939-944

14 Han C, Jo SA, Jo I et al. An adaptation of the Korean mini-mental state examination (K-MMSE) in elderly Koreans: demographic influence and population-based norms (the AGE study). Arch Gerontol Geriatr 2008; 47: 302-310

15 de Bakker PI, Yelensky R, Pe'er I et al. Efficiency and power in genetic association studies. Nat Genet 2005; 37: 1217-1223

16 Kang YW, Na DL, Hahn S. A validity study on the Korean Mini-Mental State Examination in dementia patients. J Korean Neurol Assoc 1997; 15: $300-308$

17 Folstein MF, Folstein SE, McHugh PR. "Mini-mental state". A practical method for grading the cognitive state of patients for the clinician. J Psychiatr Res 1975; 12: 189-198

18 Morris JC. The Clinical Dementia Rating (CDR): current version and scoring rules. Neurology 1993; 43: 2412-2414

19 Balding DJ. A tutorial on statistical methods for population association studies. Nat Rev Genet 2006; 7: 781-791

20 Schaid DJ, Jacobsen SJ. Biased tests of association: comparisons of allele frequencies when departing from Hardy-Weinberg proportions. Am J Epidemiol 1999; 149: 706-711

21 Barrett JC, Fry B, Maller J et al. Haploview: analysis and visualization of LD and haplotype maps. Bioinformatics 2005; 21: 263-265

22 Stephens $M$, Donnelly P. A comparison of bayesian methods for haplotype reconstruction from population genotype data. Am J Hum Genet 2003; 73: 1162-1169

23 Storey JD, Tibshirani R. Statistical significance for genomewide studies. Proc Natl Acad Sci USA 2003; 100: 9440-9445

24 Rodriguez S, Gaunt TR, Day IN. Hardy-Weinberg equilibrium testing of biological ascertainment for Mendelian randomization studies. Am J Epidemiol 2009; 169: 505-514

25 Grunblatt E, Reif A, Jungwirth $S$ et al. Genetic variation in the choline 0 -acetyltransferase gene in depression and Alzheimer's disease: the VITA and Milano studies. J Psychiatr Res 2011; 45: 1250-1256

26 Anand P, Singh B. A review on cholinesterase inhibitors for Alzheimer's disease. Arch Pharm Res 2013; 36: 375-399

27 Lee JJ, Jo SA, Park JH et al. Choline acetyltransferase 2384 G > a polymorphism and the risk of Alzheimer's disease. Alzheimer Dis Assoc Disord 2012; 26: 81-87

28 Myung W, Lim SW, Kim S et al. Serotonin transporter genotype and function in relation to antidepressant response in Koreans. Psychopharmacology (Berl) 2013; 225: 283-290 